\title{
Estudo Da Atividade Antioxidante Do Óleo Essencial De Alecrim (Rosmarinus Officinalis L) No Tratamento Ao Estresse Oxidativo Na Doença De Alzheimer
}

\section{Study of the Antioxidant Activity of Rosemary Essential Oil (Rosmarinus Officinalis L) in the Treatment of Oxidative Stress in Alzheimer's Disease}

DOI: $10.54019 /$ sesv2n3-012

Recebimento dos originais: 05/07/2021 Aceitação para publicação: 20/08/2021

\section{Letícia Grejo de Oliveira}

Graduada em Licenciatura e Bacharelado em Química Industrial pela Fundação Educacional do Município de Assis

Instituição: FEMA-Fundação Educacional do Município de Assis

Endereço: Rua Gerson Zanini, 463 - Jardim Paraíso, Cândido Mota -SP - 19880556, Brasil

E-mail: leticiagrejo@hotmail.com

\section{Mary Leiva de Faria}

Doutora em Ciências (área Química Orgânica) pela Universidade Federal de São Carlos

Instituição: FEMA-Fundação Educacional do Município de Assis

Endereço: Av. Getúlio Vargas, 1200 - Vila Nova Santana, Assis -SP - 19807-634, Brasil

E-mail:ml.faria@uol.com.br

\section{Elaine Amorim Soares}

Mestre em Ciência de Alimentos pela Universidade Estadual de Londrina Instituição: FEMA-Fundação Educacional do Município de Assis

Endereço: Av. Getúlio Vargas, 1200 - Vila Nova Santana, Assis -SP - 19807-634, Brasil

E-mail - eamorims@gmail.com

\section{RESUMO}

O óleo essencial de alecrim (Rosmarinus offininalis $L$ ) pode ser utilizado na prevenção do Alzheimer em decorrência da sua composição química, especialmente compostos terpênicos que atuam pela sua ação antioxidante ou pela inibição da acetilcolinesterase (AChE), que retarda a hidrólise catabólica da acetilcolina com o intuito de compensar a escassez da mesma nos terminas sinápticos. Esse trabalho teve o objetivo de extrair e determinar a atividade antioxidante do óleo essencial de alecrim. Primeiramente extraiu-se o óleo essencial do material botânico de alecrim por hidrodestilação em destilador do tipo Clevenger. A determinação da capacidade antioxidante foi realizada em triplicata pelo método de radical livre utilizando o reagente 2,2-difenil-1-picril hidrazil (DPPH). O rendimento obtido na extração do óleo essencial de alecrim foi de 
$0,42 \%$. O resultado obtido para a atividade antioxidante do óleo essencial de alecrim foi satisfatório, sendo a capacidade de redução de DPPH em 30 minutos de $87,21 \% \pm 0,25$. Os resultados obtidos indicam que o óleo essencial de alecrim pode ser utilizado no tratamento da doença de Alzheimer, visto que o mesmo pode atuar inibindo o stress oxidativo, a principal causa para o desenvolvimento de doenças neurodegenerativas. Deve-se considerar, entretanto, a composição do óleo essencial visto que os fatores ambientais e de cultivo podem influenciar na capacidade antioxidante do mesmo.

Palavras-Chave: Óleo essencial, Alzheimer, Alecrim.

\section{ABSTRACT}

Rosemary essential oil (Rosmarinus offininalis $L$ ) can be used in the prevention of Alzheimer's due to its chemical composition, especially terpenic compounds that act by its antioxidant action or by the inhibition of acetylcholinesterase (AChE), which delays the catabolic hydrolysis of acetylcholine in order to compensate the lack of it in the synaptic terminals. This work aimed to extract and determine the antioxidant activity of rosemary essential oil. First, the essential oil was extracted from the rosemary botanical material by hydrodistillation in a Clevenger distiller. The antioxidant capacity was determined in triplicate by the free radical method using the reagent 2,2-diphenyl-1-picryl hydrazyl (DPPH). The yield obtained from the extraction of rosemary essential oil was $0.42 \%$. The result obtained for the antioxidant activity of rosemary essential oil was satisfactory, and the DPPH reduction capacity in 30 minutes was $87.21 \% \pm 0.25$. The results obtained indicate that rosemary essential oil can be used in the treatment of Alzheimer's disease, since it can act by inhibiting oxidative stress, the main cause for the development of neurodegenerative diseases. However, the composition of the essential oil should be considered, since environmental and cultivation factors can influence its antioxidant capacity.

Keywords: Essential oil, Alzheimer's, Rosemary.

\section{INTRODUÇÃO}

O Alzheimer é uma doença neurodegenerativa progressiva e irreversível que normalmente afeta os idosos devido ao mau processamento de algumas proteínas decorrente do envelhecimento natural, porém, há casos raros sobre o aparecimento dessa enfermidade em pessoas mais jovens associada aos hábitos não saudáveis adquiridos pela população. O Alzheimer precoce tem como principal responsável o estresse oxidativo, ou seja, o desequilíbrio entre compostos antioxidantes e oxidantes, no qual favorece o excesso de radicais livres no organismo, principal responsável pela morte de células (BARBOSA et al., 
2010). Em decorrência desse desequilíbrio, o Alzheimer se desenvolve quando o ataque desses radicais livres em excesso ocorre ao nível mitocondrial (ECYCLE, 2015).

A doença de Alzheimer (DA) está relacionada também à diminuição das taxas de acetilcolina (ACh) no processo sináptico. Esta redução tem como consequência uma diminuição da neurotransmissão colinérgica cortical, bem como de outros neurotransmissores como noradrenalina, dopamina, serotonina e glutamato, surgindo as disfunções neuroquímicas e sinápticas (LIMA, 2008; DE FALCO, 2016).

Outra causa da DA, segundo a hipótese da cascata amilóide, é o aumento da quebra das ligações peptídicas da proteína precursora amiloide (APP). Esse processo, nomeado de clivagem proteolítica, tem como produto principal o peptídeo $\beta$-amilóide $(A \beta)$, que seria o responsável por iniciar uma cascata de eventos tóxicos que conduzem à formação de placas senis e a neurodegenerescência como a hiperfosforilação da Tau, que em sua forma hiperfosforilada se torna um polímero insolúvel filamentoso (GABRIEL, 2019).

Dentre os tratamentos preventivos da DA, 0 mais requerido internacionalmente em decorrência de não apresentar toxicidade ao corpo humano é o tratamento aromaterapico que consiste na aplicação de óleos essenciais extraído especialmente das plantas da família Laminaceae (ÉVORA, 2015). O óleo essencial de alecrim (Rosmarinus offininalis $L$ ) pode ser utilizado na prevenção do Alzheimer em decorrência da sua composição química, especialmente compostos terpênicos, que possuem a capacidade de estimular o sistema nervoso central e consequentemente auxiliar em melhorias ao sistema cognitivo (PORTE; GODOY, 2001). Esses compostos atuam pela sua ação antioxidante ou pela inibição da acetilcolinesterase (AChE), que retarda a hidrólise catabólica da acetilcolina com o intuito de compensar a escassez da mesma nos terminas sinápticos (OBULESU; RAO, 2011; SOUZA et al., 2012). Desta forma, esse trabalho teve o objetivo de extrair e determinar a atividade antioxidante do óleo essencial de alecrim (Rosmarinus officinalis $L$.). 


\section{METODOLOGIA}

Foram selecionadas plantas adultas da espécie de Salvia rosmarinus comumente conhecidos como alecrim, provenientes da cidade de Cândido Mota. Após a coleta foram selecionadas as folhas frescas para o os procedimentos experimentais.

Para a extração foram utilizadas $50 \mathrm{~g}$ do material botânico em $500 \mathrm{~mL}$ de água destilada submetidos a hidrodestilação em destilador do tipo Clevenger (figura 1), sob a temperatura de $150^{\circ} \mathrm{C}$ por três horas. O padrão de extração foi repetido 7 vezes totalizando $350 \mathrm{~g}$ de alecrim o que resultou em $1,5 \mathrm{~mL}$ de óleo essencial que foi armazenado em frasco âmbar esterilizado. Após a extração foi adicionado um pouco de $\mathrm{Na}_{2} \mathrm{SO}_{4}$ (sulfato de anidrido) para dessecar as gotas de água que estavam presentes no óleo. Posteriormente o recipiente foi embalado em papel alumínio e armazenado a baixas temperaturas.

\section{Figura 1 - Destilador do tipo Clevenger}

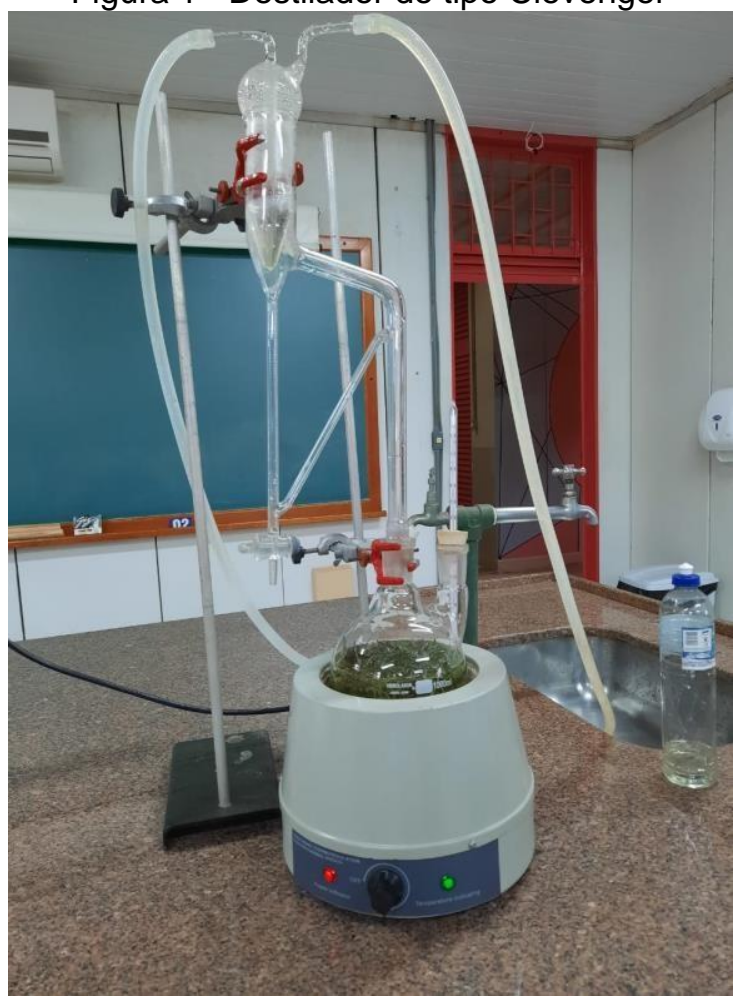

Para determinar a capacidade antioxidante foi preparada previamente a amostra do reagente 2,2-difenil-1-picril hidrazil (DPPH) na concentração de $60 \mu \mathrm{M}$, em que 2,4 mg foi dissolvida em $100 \mathrm{~mL}$ de metanol. A solução foi armazenada em frasco âmbar e envelopada em papel alumínio para o uso posterior. A leitura dessa solução foi realizada em um ambiente escuro utilizando-se cubeta de 
quartzo em espectrofotômetro ajustado para $515 \mathrm{~nm}$, em que o metanol foi utilizado como branco.

A absorbância do óleo essencial de alecrim foi feita em triplicata pelo método de radical livre, em que $0,5 \mathrm{~mL}$ do óleo essencial foi homogeneizado com 3,9 mL do radical estável DPPH, o que provocou a mudança de coloração do extrato de amarelo para roxo em razão da adição do reagente. Após o repouso de 30 minutos com a ausência de luz, a leitura foi feita em espectrofotômetro de UV a $515 \mathrm{~nm}$ utilizando o metanol como branco.

A análise da atividade antioxidante do óleo de alecrim foi calculada separadamente para cada uma das três absorbância obtidas das amostras do óleo essencial de alecrim pelo método de porcentagem de redução de DPPH, utilizando sequencialmente as equações 1 e 2 abaixo.

Eq. 1

$$
\% \mathrm{DPPH}_{\text {remanescente }}=\frac{\mathrm{Abs}_{\text {amostra }}}{\overline{\mathrm{Abs}_{\text {controle }}}} \times 100
$$

Onde:

Abs controle é a absorbância da solução metanólica do radical DPPH•

Absamostra é a absorbância do radical na presença do extrato

Eq. 2

$$
\text { \%Redução de DPPH = } 100-\% \mathrm{DPPH}_{\text {remanescente }}
$$

Posterior ao cálculo foi realizada a média de porcentagem de redução de DPHH obtidas, e foi calculado o desvio padrão das amostras pela equação 3 abaixo.

$$
\mathrm{Dp}=\sqrt{\frac{\sum\left(\mathrm{x}_{\mathrm{i}}-\overline{\mathrm{x}}\right)^{2}}{\mathrm{n}}}
$$

Onde:

Xi: valor individual da absorbância da amostra

$\dot{X}$ : valor da média da absorbância das amostras

$\mathrm{n}$ : número das amostras

\section{RESULTADOS E DISCUSSÃO}

A extração por hidrodestilação do óleo essencial de alecrim Rosmarinus officinalis em destilador do tipo Clevenger, utilizando folhas frescas do material botânico, forneceu um rendimento de $0,42 \%$. O rendimento do óleo foi calculado 
pela quantidade de óleo que se obteve com a massa de vegetal usada. Obtevese 1,47 gramas de óleo a partir de 350 gramas de folhas frescas de alecrim.

O resultado obtido foi próximo ao obtido por Rocha (2016), o qual aborda que o padrão de rendimento da extração por hidrodestilação do alecrim é de $0,59 \%$.

Segundo Cutrim et al. (2019), o óleo essencial apresenta maior rendimento quando extraído em solventes orgânicos, porém no mesmo artigo os autores afirmam que rendimento máximo é de $1 \%$. $O$ autor saliente ainda que a utilização de folhas secas também resulta em um maior percentual de extração do óleo essencial.

O óleo essencial de alecrim apresentou densidade menor que a da água, cheiro forte e característico do alecrim, bem como coloração branca (quase incolor) logo no início da extração, tornando-se amarelada, cor característica do material, com o decorrer do tempo, conforme citado por Pala et al. (2010). Essa variação de coloração pode ser observada na figura 2 .

Figura 2 - Óleo essencial de Alecrim (Rosmarinus officinalis) no início (A) e final (B) da extração.
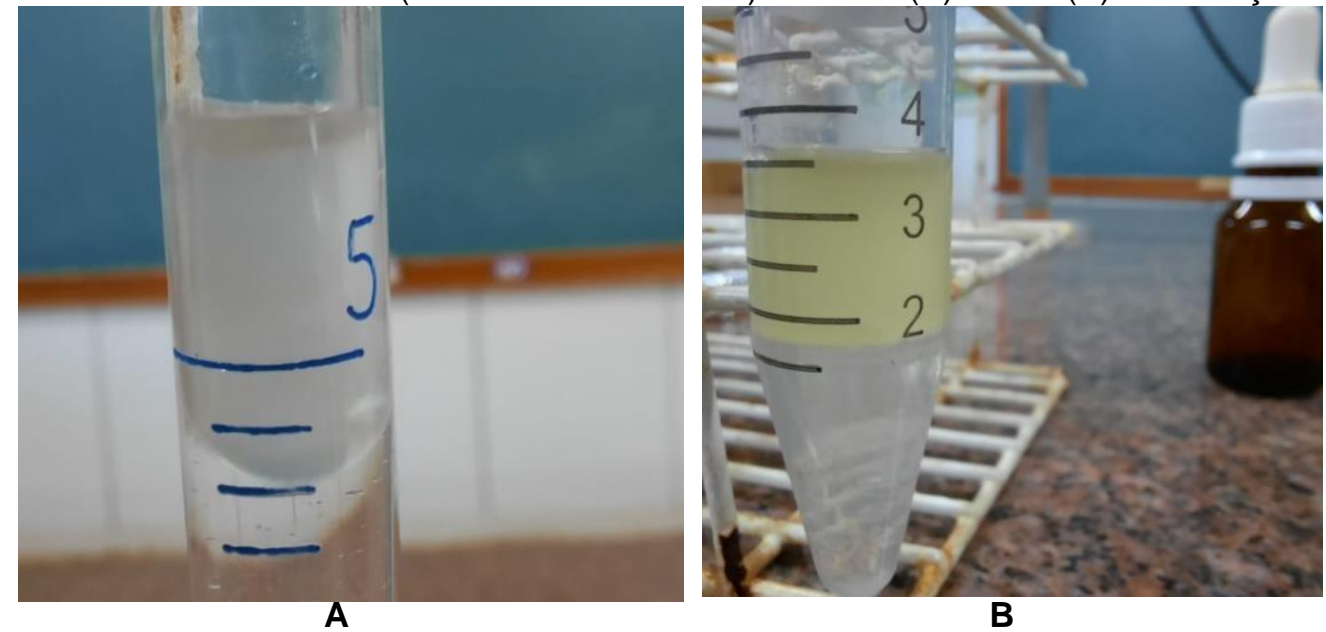

O valor médio das três absorbâncias das amostras de óleo de alecrim para determinação da atividade antioxidante foi de 0,1047. A atividade antioxidante total, calculada pela porcentagem de redução de DPPH em 30 minutos, foi de $87,21 \% \pm 0,25$.

Segundo Cutrim et al. (2019), o percentual máximo de inibição do óleo essencial de alecrim (Rosmarinus. Officinalis L.) em solução alcoólica é de 99,8\% em concentração elevada. $\mathrm{O}$ resultado obtido no presente trabalho mostrou-se satisfatório, inclusive se comparado à atividade antioxidante dos óleos essenciais 
do capim limão e da erva cidreira, utilizados no tratamento da doença de Alzheimer. Silva et al. (2017) analisaram, também pelo método de DPPH, as espécies de Cymbopogon citratus, conhecida como capim limão e Melissa officinalis, comumente conhecida como erva-cidreira. O capim limão apresentou atividade antioxidante total de $20,90 \%$ e a erva-cidreira de $78,12 \%$.

\section{CONCLUSÃO}

A extração do óleo essencial de alecrim (Rosmarinus officinalis L.) por hidrodestilação, com a utilização das folhas frescas, foi muito próximo ao descrito na literatura.

O resultado da capacidade antioxidante do óleo essencial de alecrim obtido por hidrodestilação foi de $87,21 \% \pm 0,25$, um resultado satisfatório comprovando a potencialidade referente às espécies da família de Laminaceae.

Em relação à capacidade antioxidante, os resultados obtidos indicam que o óleo essencial de alecrim pode ser utilizado no tratamento da doença de Alzheimer, visto que o mesmo pode atuar inibindo o stress oxidativo, a principal causa para o desenvolvimento de doenças neurodegenerativas.

Deve-se considerar, entretanto, a composição do óleo essencial visto que os fatores ambientais e de cultivo podem influenciar na capacidade antioxidante do mesmo. 


\section{REFERÊNCIAS}

BARBOSA, Kiriaque Barbosa; COSTA, Neuza Maria; ALFENAS, Rita de Cássia; DE PAULA, Sérgio Oliveira; MINIM, Valéria Paula; BRESSANI, Josefina. Estresse oxidativo: conceito, implicações e fatores modulatórios. Revista de nutrição. Campinas, v. 23, $n^{\circ} 4,2010$, p. 629-643.

CUTRIM, E. S. M.; TELES, A. M.; MOUCHREK, A. N.; MOUCHREK Filho, V. E.; EVERTON, G. O. Avaliação da Atividade Antimicrobiana e Antioxidante dos Óleos Essenciais e Extratos Hidroalcoólicos de Zingiber officinale (Gengibre) e Rosmarinus officinalis (Alecrim). Revista Virtual de Química. v. 11, o․ 1., 2019, p. 60-81.

DE FALCO, Anna; CUKIERMAN, Daphne S.; HAUSER-DAVIS, Rachel A.; REY, Nicolás A. Doença de Alzheimer: hipóteses e perspectivas de tratamento. Química Nova. São Paulo, v. 39, no. 1, 2016, p. 63-80.

ECYCLE, E. Antioxidantes: o que são e em quais alimentos encontrá-los. 2015. Disponível em: <https://www.ecycle.com.br/antioxidantesantioxidante/\#: :text=A\%20no\%20organismo.-

,Lista\%20de\%20alimentos\%20ricos\%20em\%20antioxidantes\%20beta\%2Dcarot eno\%20e\%20licopeno,como\%20br\%C3\%B3colis\%2C\%20ervilha\%20e\%20espin afre >. Acesso em: 06 jun. 2020.

ÉVORA, Leisa Nélida Pinto. Actividades biológicas e citotoxidade do óleo essencial de Rosmarinus officinalis L. 2015. 94p. Dissertação (Mestrado) Universidade de Coimbra, 2015.

GABRIEL, António José dos Santos. Avaliação do sistema colinérgico na doença de Alzheimer. 2019, 187p. Tese de doutorado em Biociências especializado em Biologia Celular e Molecular - Universidade de Coimbra, 2019.

LIMA, Daniele A. Tratamento Farmacológico da Doença de Alzheimer. Revista do Hospital Universitário Pedro Ernesto. 2008, p.1-10.

OBULESU, M.; RAO, Dowlathabad Muralidhara. Efeito de extratos de plantas na doença de Alzheimer: uma visão dos caminhos terapêuticos. PubMed - J Neurosci Rural Pract. 2011, p. 56-61

PALA, Amanda Caroline Tomé; SALIN, Camila Tozzi; CORTEZ, Lúcia Elaine Ranieri. Controle de qualidade de óleos essenciais de alecrim (Rosmarinum officinalis) e lavanda (lavandula augustifolia) comercializados em farmácias de dispensação. Mostra Interna de Trabalhos de Iniciação Científica. 2010, p.4.

PORTE, Alexandre; GODOY, Ronoel Luiz de Oliveira. Alecrim (Rosmarinus Officinalis L.): Propriedades Antimicrobiana e Química do Óleo Essencial. B.CEPPA. Curitiba, v. 19, ํㅡ. 2, 2001, p. 193-210.

ROCHA, Joselayne Silva; VASCONCELOS, Tatiana Cristina. Dificuldades de aprendizagem no ensino de química: algumas reflexões. XVIII Encontro Nacional de Ensino de Química (XVIII ENEQ) Florianópolis, SC, 2016. 
SILVA, Nathalia Lucca; ARAÚJO, Ítalo Péricles Caíque; BATISTA, Marília Renata Ferreira; SANTOS, Thaís Bruna Aquino; FERNANDO, Walem Luiz; AMARAL, Fabrício Rodrigues. Determinação da atividade antioxidante e do teor de flavonoides totais equivalentes em quercetina em extrato aquoso de folhas de Cymbopogon citratus (d.c.) stapf e Melissa officinalis lam obtidos por decocção.

Conexão Ci. Minas gerais. v.12, no. 1., 2017, p. 46 - 53.

SOUZA, Stefânia P. de; VALVERDE, Simone S.; SILVA, Raphael L.N.R. da; LIMA, Keila S.C.; LIMA, Antônio L.S. Óleos essenciais como inibidores da acetilcolinesterase. Revista Fitos. v.7, nำ.4, 2012, p. 259-265 80X. 